\title{
Correction to: Metabolomics profiles of patients with Wilson disease reveal a distinct metabolic signature
}

\author{
Gaurav V. Sarode ${ }^{1} \cdot$ Kyoungmi Kim $^{2} \cdot$ Dorothy A. Kieffer $^{1} \cdot$ Noreene M. Shibata $^{1} \cdot$ Tomas Litwin $^{3}$. \\ Anna Czlonkowska ${ }^{3} \cdot$ Valentina Medici ${ }^{1}$ (I)
}

Published online: 3 December 2019

(c) Springer Science+Business Media, LLC, part of Springer Nature 2019

\section{Correction to: Metabolomics (2019) 15:43 https://doi.org/10.1007/s11306-019-1505-6}

In the originally published version of this article, there was an error.

The metabolomics platform used for the analysis is GCTOF-MS, Gas Chromatography Time-of-Flight Mass Spectrometry and not Hydrophilic Interaction Liquid Chromatography-Quadrupole Time of Flight Mass Spectrometry as indicated in the original version.

The wrong methodology is reported in 4 points:
1. Abbreviations

2. Abstract

3. Subjects and Methods

4. Discussion

The authors apologize for the mistake which does not affect the conclusions.

Publisher's Note Springer Nature remains neutral with regard to jurisdictional claims in published maps and institutional affiliations.

The original article can be found online at https://doi.org/10.1007/ s11306-019-1505-6.

Valentina Medici

vmedici@ucdavis.edu

1 Division of Gastroenterology and Hepatology, Department of Internal Medicine, University of California Davis, $4150 \mathrm{~V}$ Street, Suite 3500, Sacramento, CA 95817, USA

2 Division of Biostatistics, Department of Public Health Sciences, University of California Davis, Davis, CA, USA

3 Department of Neurology, Institute of Psychiatry and Neurology, Warsaw, Poland 